\title{
Prevalence of Macroprolactinemia in Patients with Hyperprolactinemia Using Roche Elecsys Platform in a Large Tertiary Referral Center in UAE
}

\author{
Abdulla Al Nuaimi ${ }^{a}$ Raya Almazrouei ${ }^{b}$ Yusra Othman ${ }^{c}$ Salem Beshyah ${ }^{\text {d, e }}$ \\ Khaled M. Aldahmani ${ }^{b}$ f \\ aDivision of Endocrinology, Sheikh Khalifa Medical City, Abu Dhabi, UAE; 'Division of Endocrinology, Tawam Hospital, Al

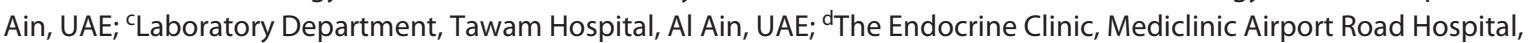 \\ Abu Dhabi, UAE; ${ }^{D}$ Department of Medicine, Dubai Medical College, Dubai, UAE; ${ }^{\text {DDepartment }}$ of Medicine, UAE University, \\ Al Ain, UAE
}

\section{Keywords}

Hyperprolactinemia · Macroprolactin · Pituitary

\begin{abstract}
Objectives: Macroprolactin (macroPRL) excess is an important cause of hyperprolactinemia. Several prolactin assays have high reactivity to macroPRL. However, macroPRL screening is not routinely performed in many labs. This study aimed to evaluate the prevalence of macroprolactinemia (MP) in patients with elevated prolactin using the Roche Elecsys assay in a large tertiary center in UAE. Materials and Methods: Consecutive samples of patients with elevated prolactin presenting to Tawam Hospital from June to August 2018 were evaluated for MP. Polyethylene glycol (PEG) was used to precipitate macroPRL. Monomeric prolactin recovery cutoff $\leq 50 \%$ was used to determine the prevalence of MP. Results: A total of 180 patients with elevated prolactin were included in the study with a mean age of $33.1 \pm 11.9$ years. The majority were women (87.2\%), and about $77.8 \%$ were newly diagnosed patients with hyperprolactinemia. The main indications for prolactin testing were menstrual irregularity $(n=121)$, infertility $(n=11)$, galactorrhea $(n=11)$, and sellar masses $(n=12)$. MP was present in $8.3 \%$ of the pa-
\end{abstract}

karger@karger.com www.karger.com/dde

Karger!

GOPEN ACCESS
(C) 2021 The Author(s)

Published by S. Karger AG, Basel

This is an Open Access article licensed under the Creative Commons Attribution-NonCommercial-4.0 International License (CC BY-NC) (http://www.karger.com/Services/OpenAccessLicense), applicable to the online version of the article only. Usage and distribution for commercial purposes requires written permission. tients. The median (IQR) of total prolactin level was 740.5 $(579-1,085) \mathrm{IU} / \mathrm{m}$ before PEG precipitation and was not significantly different between MP and true hyperprolactinemia cases. Three patients with MP had pituitary MRI evaluation, which was normal. Eight patients with MP were treated with cabergoline. Conclusion: One in 12 patients with hyperprolactinemia had MP on the Roche Elecsys assay defined as the cutoff recovery of $\leq 50 \%$ post-PEG precipitation. Physicians should be aware of the prolactin assay used in their labs, and we recommend routine macroPRL assessment in mild hyperprolactinemia samples in labs using the Roche Elecsys platform.

(C) 2021 The Author(s).

Published by S. Karger AG, Basel

\section{Introduction}

Hyperprolactinemia is a common disorder with increasing prevalence over the last few decades [1]. It is more common in women and typically presents with symptoms related to hypogonadism [2]. Hyperprolactinemia can be due to physiological, pharmacological, or pathological causes [2]. Macroprolactin (macroPRL) is a large prolactin molecule mostly due to prolactin/immu- 
noglobulin complexes. Macroprolactinemia (MP) is an important cause of hyperprolactinemia and occurs when macroPRL constitutes the majority of total prolactin in the circulation [3]. It is usually suspected in patients with idiopathic or asymptomatic hyperprolactinemia [4].

Some prolactin assays have low reactivity to macroPRL and reported high prolactin represents true hyperprolactinemia, while other assays overestimate the prolactin level due to strong reactivity to macroPRL [5]. In a recent study of the etiologies of hyperprolactinemia, we did not observe macroPRL as an important cause of elevated prolactin using the Beckman prolactin assay [6]. This particular essay is known to have low reactivity to macroPRL [5]. However, in 2018, our center introduced the Roche Elecsys platform for prolactin assessment. The data in the literature regarding the reactivity of this assay to macroPRL are not consistent $[5,7]$. We wished to evaluate the frequency of MP in patients with elevated prolactin using the Roche Elecsys assay in a large tertiary center in the UAE.

\section{Subjects and Methods}

Data were collected for consecutive patients with elevated prolactin who underwent testing for MP at Tawam Hospital for 3 months (June-August 2018). In addition, demographic and clinical data were collected including age at the time of prolactin assessment, gender, ethnicity, the indication for prolactin request, prolactin level pre- (total) and post-polyethylene glycol (PEG) precipitation (mIU/L, convert from mcg by multiplying 21.3), estradiol/testosterone level, and LH/FSH if available within 1 week around the prolactin measurement. Furthermore, we recorded whether patients underwent pituitary imaging and/or commenced on dopamine agonist (DA) therapy.

Blood samples were collected randomly during the regular working hours (08:00-20:00) regardless of the feeding status. Serum prolactin was measured using Roche Cobas e 801 sandwich electrochemiluminescence binding immunoassay (Roche Diagnostics, Mannheim, Germany). The assay is calibrated against the World Health Organization Third International Standard for prolactin (84/500). The assay's analytical sensitivity in our laboratory was $<2 \mu \mathrm{IU} / \mathrm{mL}$, and the coefficient of variation was $5 \%$. The normal value is $86-324 \mu \mathrm{IU} / \mathrm{mL}$ for men and $102-496 \mu \mathrm{IU} / \mathrm{mL}$ for nonpregnant women. MacroPRL and oligomers were precipitated using $25 \%$ aqueous PEG solution (ratio $1+1$ ). After centrifugation, the supernatant containing monomeric prolactin is used to measure prolactin the same way as a native sample. Monomeric prolactin recovery cutoff $\leq 50 \%$ was used to determine the prevalence of MP [8].

Proportional outcomes were presented as numbers and percentages. Continuous values were expressed as mean with standard deviation or median with interquartile range if normally or non-normally distributed respectively We performed $\chi^{2}$ and Fisher's exact tests for categorical variables, $t$ tests for normally distributed variables, and the Mann-Whitney $U$ test for non-normally distributed variables. A 2 -tailed probability value of $<5 \%$ was considered statistically significant. Statistical analysis was performed using statistical software (Stata13, Stata Corp LP, College Station, TX, USA).

\section{Results}

A total of 180 patients with hyperprolactinemia were included in the study (Table 1). The mean age of the patients was $33.1 \pm 11.9$ years. The majority were women (87.2\%), and $68.9 \%$ were UAE national. One hundred and forty patients $(77.8 \%)$ were newly evaluated patients with hyperprolactinemia. Overall, the most common indications for prolactin assessment were menstrual irregularities $(n=121,67.2 \%)$, infertility $(n=11,6.1 \%)$, galactorrhea $(n=11,6.1 \%)$, and sellar masse/lesion $(n=12,6.7 \%)$.

For men, the four most common indications were sellar masses $(n=5)$, infertility $(n=3)$, erectile dysfunction $(n=3)$, and gynecomastia $(n=2)$. The majority of the patients (71\%) did not have pituitary MRI at data analysis time. Of those with imaging, 28 (15.6\%) had normal findings, $14(7.8 \%)$ had microadenoma, and $10(5.6 \%)$ had macroadenoma.

No specific cause was identified in $52.2 \%$ of the cases, while polycystic ovarian syndrome (PCOS), antipsychotic mediation, physiological reasons, and hypothalamic-pituitary axis-related conditions were the reported causes in $22.8 \%, 6.7 \%, 4.4 \%$, and $13.9 \%$ of cases, respectively. Almost one-third (35\%) of the patients were started on DA therapy.

Based on the macroPRL recovery cutoff $\leq 50 \%$ postPEG precipitation, only 15 out of 180 (8.3\%; 95\% CI $4.7-$ $13.4 \%)$ patients had MP. The rate of MP was $5 \%$ and $11.7 \%$ when the macroPRL recovery of $\leq 40 \%$ and $\leq 60 \%$ was used, respectively. The median (IQR) of the total prolactin level for the whole cohort was $740.5(579-1,085)$ $\mathrm{IU} / \mathrm{m}$ before PEG precipitation, which was not significantly different between patients with MP compared to those with true hyperprolactinemia (Table 1; Fig. 1).

Estradiol and testosterone levels were available for few patients only in the MP group, and therefore, the comparison between the 2 groups was not possible. LH and FSH levels were available for 4 female patients with MP and 98 and 100 female patients with true hyperprolactinemia. There was no statistically significant difference between the 2 groups in terms of LH and FSH levels (Table 1).

Only 3 patients with MP had pituitary MRI imaging; all were normal. Menstrual irregularities were the main reason for prolactin testing in both groups. Like in the true hyperprolactinemia group, most MP patients had 
Table 1. Comparison between patients with MP versus true hyperprolactinemia based on the recovery percentage $\leq 50 \%$

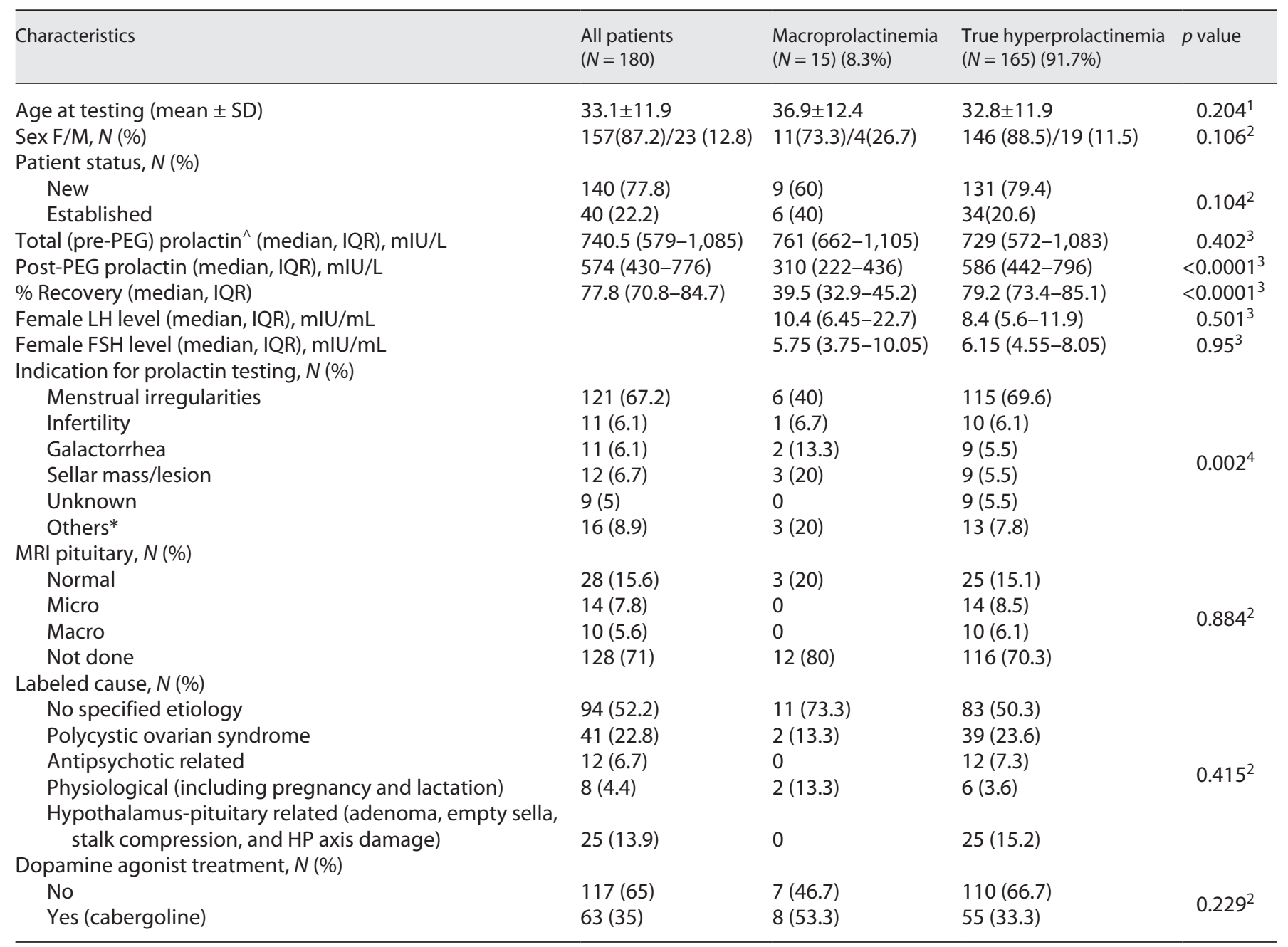

SD, standard deviation; PEG, polyethylene glycol; IQR, interquartile range; MP, macroprolactinemia. ^ Normal prolactin range: females: 104-496 mIU/L, males:86-324 mlU/L. * Others in the macroprolactinemia group included: 1 post-craniopharyngioma, 1 erectile dysfunction, and 1 hypotension. Others in the true hyperprolactinemia group included: 1 seizure, 1 traumatic pituitary damage, 1 menorrhagia, 2 hirsutism, 2 gynecomastia, 2 ED, 1 dysmenorrhea, 1 hypogonadism, $1 \mathrm{GH}$ deficiency, and 1 decreased libido. $p$ value derived from ${ }^{1} t$ test. ${ }^{2}$ Fisher's exact test. ${ }^{3}$ Mann-Whitney $U$ test. ${ }^{4} X^{2}$ test.

no specified etiology for hyperprolactinemia: 83 (50.3\%) and $11(73.3 \%)$, respectively. Cabergoline was used in 8 (53.3\%) patients with MP and in $55(33.3 \%)$ patients with true hyperprolactinemia (Table 1 ).

\section{Discussion}

The prevalence of MP in our study was $8.3 \%$. In a meta-analysis of 67 studies, the overall prevalence of MP was $19 \%$ but varied from 0 to $56 \%$ among the different studies
[9]. This wide variability could largely be attributed to the difference in prolactin assays used in these studies. In one study using different prolactin assays in people with MP, the prolactin level was highest when assessed by the Roche Elecsys assay compared to other prolactin assays [7]. For example, the prolactin level using the Roche Elecsys assay was 2-5 folds higher than Bayer centaur and Beckman Access, which had the lowest reactivity to macroPRL [7]. Besides, other factors such as study population and the threshold at which macroPRL excess is defined could also impact the prevalence of MP. In addition, the prolactin 


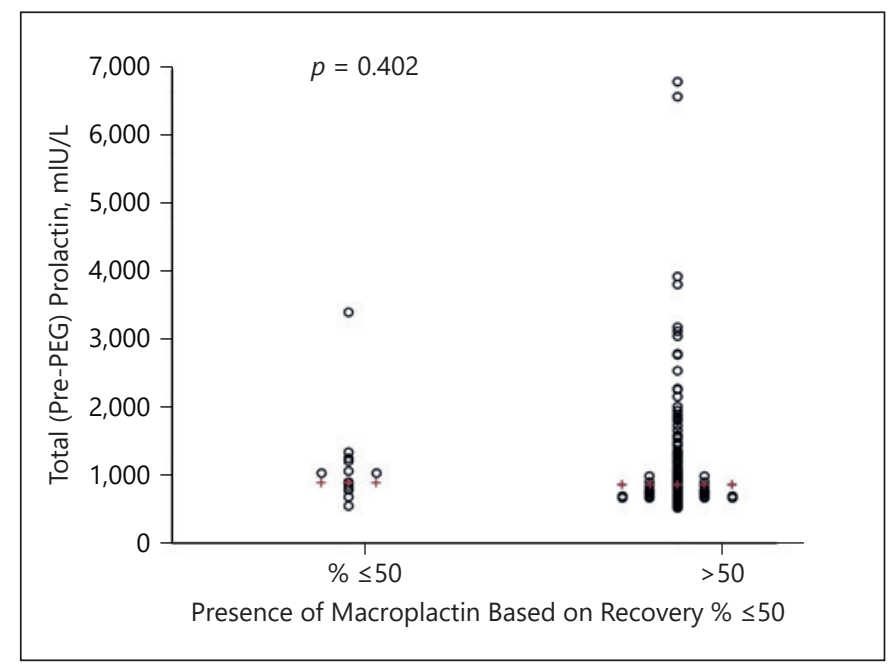

Fig. 1. Distribution of total prolactin level according to the presence of macroPRL based on the recovery $\% \leq 50$. Red crosses represent the median. $p$ value derived from the Mann-Whitney test. PEG, polyethylene glycol; macroPRL, macroprolactin.

recovery cutoff used affects the prevalence of hyperprolactinemia. In this study, the rate of MP varies between $5 \%$ and $11.7 \%$ when the macroPRL recovery of $\leq 40 \%$ and $\leq 60 \%$ was used, respectively.

It is believed that macroPRL has no or reduced bioactivity in vitro; people with MP may not present with typical hyperprolactinemia symptoms [5]. However, in our study, about $40 \%$ of patients with MP had menstrual irregularities, while galactorrhea was less common (13\%) (rates for menstrual irregularities and galactorrhea in true hyperprolactinemia were $69 \%$ and $5 \%$, respectively). Similarly, several studies have shown a high frequency of reproductive symptoms (15-80\% menstrual disturbance, $0-46 \%$ galactorrhea) in MP [10-13]. Therefore, symptoms of reproductive dysfunction might overlap between MP and true hyperprolactinemia cases, and differentiation between the two could be challenging based on the clinical assessment alone. Also, it is crucial to keep in mind the possible presence of other comorbidities such as PCOS as a cause of reproductive dysfunction in those with MP. Nonetheless, Kalsi et al. [14], in a recent study, showed that MP was the potential cause of reproductive dysfunction in about half of patients in whom other confounding factors were carefully evaluated. Similarly, only two patients in our MP cohort were diagnosed with PCOS. Among the male patients in the study, the indications for prolactin testing were variable with no clear dominance of particular symptoms.

Prevalence of Macroprolactinemia
In the present study, the prolactin level was not a predictor for MP, which is in line with the current literature [15]. Several studies showed that estradiol and LH levels were higher in the MP cohort than those with true hyperprolactinemia $[16,17]$. In the present study, LH was numerically higher in the MP cohort but did not reach statistical significance, likely due to the small number of undergoing LH evaluation. Nonetheless, normal estradiol and LH levels in the setting of hyperprolactinemia might serve as a clue for the presence of MP.

MP is not associated with serious underlying etiologies and therefore requires no further investigation or treatment. In our study, $20 \%$ of the MP cohort underwent pituitary imaging (all normal), and about $50 \%$ were treated with DAs. In line with this, several studies reported increased the utilization of pituitary MRI (up to 93\%) and DA therapy $(22-72 \%)$ in this population $[10,12,13,18]$. In addition to the cost of unnecessary imaging and frequent follow-up assessment, there are considerable patient's anxiety and risk of adverse events associated with DA treatment if MP was not accurately diagnosed. To avoid these issues, it is vital for physicians to discuss with their colleagues in biochemistry labs the best strategy to investigate hyperprolactinemia cases and whether routine macroPRL is necessary.

None of the patients in our study had MP in coexistence with other causes of true hyperprolactinemia. However, abnormal pituitary imaging results ranging from empty sell to macroadenoma were reported in many patients with MP [15]. As such, physicians should also assess the level of monomeric prolactin post-PEG precipitation regardless of whether or not MP was present. Those with elevated monomeric prolactin need to be approached a similar way to actual hyperprolactinemia cases.

Our study has some limitations. First, this is a singlecenter study using the Roche Elecsys prolactin assay, and our findings may not be generalizable to data from nontertiary referral centers or labs using different prolactin platforms. Second, MP was not confirmed using gel filtration chromatography, a gold standard of macroPRL determination. Nonetheless, we used a prolactin recovery cutoff of $\leq 50 \%$ post-PEG precipitation in the Roche Elecsys, which was validated to detect macroPRL excess in all cases assessed by gel filtration chromatography [8]. Using the different recovery cutoff when evaluating for macroPRL can underestimate the coexistence of true hyperprolactinemia and properly estimation of prolactin level in post-PEG using the post-PEG reference interval would be a better option. At this point, there are no data on the 
normal range of monometric prolactin post-PEG precipitation using the Roche Elecsys assay in our population, and therefore, the coexistence of true hyperprolactinemia could have been underestimated.

In summary, MP is present in $8 \%$ of our patients with hyperprolactinemia using the Roche Elecsys prolactin assay, and we recommend routine macroPRL assessment using this platform. Further studies are needed to evaluate monomeric prolactin post-PEG treatment's normal reference range to precisely identify the coexistence of true hyperprolactinemia.

\section{Acknowledgement}

We would like to thank Mr. Omran Darwish for supervising macroprolactin testing.

\section{Statement of Ethics}

The Ethical Committee at Tawam Hospital in $\mathrm{Al}$ Ain district approved the study (MF 2058-2019-649) and consent was waived.

\section{Conflict of Interest Statement}

The authors declare no conflicts of interest that pertain to this work.

\section{Funding Sources} body.

This review paper received no specific grant from any funding

\section{Author Contributions}

All authors contributed to the conception of the study, data acquisition, and analysis, and to drafting and revising of the manuscript. They all reviewed the final manuscript.

\section{Data Availability Statement}

The data that support the findings of this study are not publicly available due to technical depository reasons but are available from the corresponding author upon reasonable request.

\section{References}

1 Soto-Pedre E, Newey PJ, Bevan JS, Greig N, Leese GP. The epidemiology of hyperprolactinaemia over 20 years in the Tayside Region of Scotland: the Prolactin Epidemiology, Audit and Research Study (PROLEARS). Clin Endocrinol. 2017;86:60-7.

2 Aldahmani KM, AlMalki MH, Beshyah SA. A rational approach to the evaluation and management of patients with hyperprolactinemia. Ibnosina J Med Biomed Sci. 2020;12:90-7.

3 Shimatsu A, Hattori N. Macroprolactinemia: diagnostic, clinical, and pathogenic significance. Clin Dev Immunol. 2012;2012:167132. Epub 2012 Dec 4. PMID: 23304187; PMCID: PMC 3529459.

4 Melmed S, Casanueva FF, Hoffman AR, Kleinberg DL, Montori VM, Schlechte JA, et al. Diagnosis and treatment of hyperprolactinemia: an Endocrine Society clinical practice guideline. J Clin Endocrinol Metab. 2011; 96:273-88.

5 Fahie-Wilson M, Smith TP. Determination of prolactin: the macroprolactin problem. Best Pract Res Clin Endocrinol Metab. 2013;27: $725-42$.

6 Malik AA, Aziz F, Beshyah SA, Aldahmani KM. Aetiologies of hyperprolactinaemia: a retrospective analysis from a tertiary healthcare centre. Sultan Qaboos Univ Med J. 2019; 19:e129-34

7 Smith TP, Suliman AM, Fahie-Wilson MN, McKenna TJ. Gross variability in the detection of prolactin in sera containing big big prolactin (macroprolactin) by commercial immunoassays. J Clin Endocrinol Metab. 2002 Dec;87(12):5410-5.

8 Glison G, Scmit P, Thix J, Hoffman JP, Humbel RL. Prolactin Results for samples containing macroprolactin are method and sample dependent. Clin chem. 2001 Feb;47(2):331-3.

9 Che Soh NAA, Yaacob NM, Omar J, Mohammed Jelani A, Shafii N, Tuan Ismail TS, et al. Global prevalence of macroprolactinemia among patients with hyperprolactinemia: a systematic review and meta-analysis. Int J Environ Res Public Health. 2020 Nov 6;17(21):8199.

10 Fahie-Wilson MN, Soule SG. Macroprolactinaemia: contribution to hyperprolactinaemia in a district general hospital and evaluation of a screening test based on precipitation with polyethylene glycol. Ann Clin Biochem. 1997 May;34(Pt 3):252-8.

11 Leslie H, Courtney CH, Bell PM, Hadden DR, McCance DR, Ellis PK, et al. Laboratory and clinical experience in 55 patients with macroprolactinemia identified by a simple polyethylene glycol precipitation method. J Clin Endocrinol Metab. 2001;86(6):2743-6.

12 Olukoga AO, Kane JW. Macroprolactinaemia: validation and application of the polyethylene glycol precipitation test and clinical characterization of the condition. Clin Endocrinol. 1999 Jul;51(1):119-26.

13 Vallette-Kasic S, Morange-Ramos I, Selim A, Gunz G, Morange S, Enjalbert A, et al. Macroprolactinemia revisited: a study on 106 pa- tients. J Clin Endocrinol Metab. 2002;87(2): 581-8.

14 Kalsi AK, Halder A, Jain M, Chaturvedi PK, Sharma JB. Prevalence and reproductive manifestations of macroprolactinemia. Endocrine. 2019 Feb;63(2):332-40. Epub 2018 Sep 29. PMID: 30269265.

15 Samson SL, Hamrahian AH, Ezzat S; AACE Neuroendocrine and Pituitary Scientific Committee, American College of Endocrinology (ACE). American Association of Clinical Endocrinologists, American College of Endocrinology disease state clinical review: clinical relevance of macroprolactin in the absence or presence of true hyperprolactinemia. Endocr Pract. 2015 Dec;21(12):1427-35. PMID: 26642103.

16 Suliman AM, Smith TP, Gibney J, McKenna TJ. Frequent misdiagnosis and mismanagement of hyperprolactinemic patients before the introduction of macroprolactin screening: application of a new strict laboratory definition of macroprolactinemia. Clin Chem. 2003 Sep;49(9):1504-9.

17 Gibney J, Smith TP, McKenna TJ. The impact on clinical practice of routine screening for macroprolactin. J Clin Endocrinol Metab. 2005 Jul;90(7):3927-32.

18 Strachan MW, Teoh WL, Don-Wauchope AC, Seth J, Stoddart M, Beckett GJ. Clinical and radiological features of patients with macroprolactinaemia. Clin Endocrinol. 2003; 59:339-46. 